\title{
MOTOR IMAGERY: PERCEPTION OR ACTION?
}

\author{
JOHN ANNETT \\ Department of Psychology, University of Warwick, Coventry CV4 7AL, U.K.
}

(Received 5 June 1994; accepted 30 November 1994)

\begin{abstract}
Motor imagery has been studied using subjective, behavioural and physiological methods and this paper reviews theoretical and practical issues from all three viewpoints. Attempts to measure motor imagery on a subjective scale have met with limited success but alternative methods are proposed. Research on mental practice suggests a number of different processes may be needed to explain the variety and variability of effects obtained. Recent studies of spatial and motor working memory signify the importance of a primarily visuo-spatial component in which actions are consciously represented together with a more properly motoric component which must be activated to generate either images or overt actions. Finally the question of whether motor imagery is primarily perceptual or motoric in character does not have a simple neurophysiological answer due to the highly distributed nature of motor control. Nevertheless some of the key mechanisms serving both spatial and motoric components have been provisionally identified.
\end{abstract}

Key Words: motor imagery; individual differences; mental practice; working memory; EEG; ERP.

\section{INTRODUCTION}

Images have frequently been conceptualised as 'pictures in the mind', as objects in the phenomenal world which may be treated in certain respects like data originating from physically present objects, as quasi sensations which are experienced, evaluated, reacted to and committed to memory like other sensory events. Motor imagery, however, would seem to involve a further, perhaps crucial, ingredient, namely voluntary control on the part of the imager as agent which goes beyond simply attending to an object of consciousness. The varieties of imaginary action include both the voluntary manipulation of imaginary objects and the imaginary manipulation of physically present objects, controlled progression through imaginal space and imaginal variations in body posture.

Several varieties of motor imagery can be distinguished. In the simplest form the subject imagines self-performed action, with 'interior' view. Alternatively the subject imagines seeing himself or another performing actions with an exterior view (sometimes called 'mental video'). Rather different is the situation in which the subject controls images of external objects, for example generates or rotates an image, or moves attention between different parts of an image, as in scanning. These types of imagery all involve voluntary action but may not involve identical cognitive processes. For example the first may involve quasi-kinaesthetic experiences whilst the second and third case may be primarily visual or spatial in character. All, however, appear to involve some degree of voluntary control and to this extent deserve the designation 'motor'.

The feeling of voluntariness, of being in control, may be attributed to the imagined actor. Shepard [85] refers to the subjective effortfulness of imagined transformations, such 
as mental rotations which are distinguishable from perceived motion and indeed it is generally postulated that these internal manipulations partake of at least some of the properties of overt voluntary action and in all probability some of the same brain mechanisms. Paivio [70, p. 72] also asserts "all mental transformations engage motor processes that derive originally from active manipulation of the referent objects ...", thus endorsing a basic postulate guiding imagery research, namely that imaging is functionally equivalent to and may share some of the brain processes associated with normal perception. By the same token a basic question concerning motor imagery is whether imaginary actions share some of the neural processes which serve overt action. This paper addresses the problem of what these mechanisms might be and what evidence we have to support the notion of processes shared by real and imaginary actions. The evidence relevant to this question comes from a variety of sources including individual differences, cognitive psychology, motor and sport psychology, cognitive neuropsychology and psychophysiology.

\section{METHODOLOGICAL ISSUES}

Questions about motor imagery have been approached through a variety of methodologies each with its own limitations but each with something to contribute to the overall perspective. The classical methodology simply requires the 'observer' to imagine a self-performed action or an external view of another actor, for instance the instruction to "imagine yourself/someone else reaching for an object on a high shelf". This is the approach taken by Galton [36] and Betts [15] and recently by Hall et al. [41] and by Isaac et al. [46] in self report imagery questionnaires when the dependent variable is usually a vividness rating. A variant on this method is to instruct the subject to mentally execute or covertly rehearse an action and this is typical of the research tradition known as 'mental practice' which is commonly used by athletes and has been extensively researched $[26,27,32,65,69]$. In this method subjects are only rarely required to report on their subjective experiences but, by contrast, the effectiveness of the procedure is assessed by reference to a performance measure, usually some enhancement of speed or accuracy which is comparable to that which would be achieved by overt practice of the same actions. An important variant on this direct method is to record physiological changes following the imagery instruction, for example EMG, EOG, heart rate and other somatic responses, or brain activity using EEG, PET, MRI, etc.

The instruction to imagine action attempts the direct manipluation of imagery but a second major group of techniques may be regarded as an indirect methodology. In these the subject is given a task believed to depend on imaginal processes and some aspect of the response is taken as the dependent variable. The mental rotation task [18] which may possibly involve motor processes is an example of this paradigm and others such as the Brooks Matrix task $[10,20]$ used to investigate short-term memory fall into the same general category insofar as performance is presumed to depend on underlying processes of image generation, manipulation and retention. Another example is to ask the subject questions such as "tell me in as much detail as you can how to tie two ends of string together to make a bow" $[3,6]$ which provides a verbal protocol with other responses such as eye movements, hand gestures and brain activity which may be taken as indicators of presumed imagery processes. The short-term memory paradigm where the to-be-remembered items are actions $[88,89]$ is a related method insofar as it also 
requires the experimental subject not only to encode but to maintain an imaginal representation, although here the dependent variable is of course items recalled, usually by reproduction.

Each of the techniques outlined above has certain possibilities and limitations. First, in all those techniques which rely principally on instructions to imagine actions the subjects' compliance with instructions cannot be assured. Where all the subject has to do is report a vividness rating the demand characteristics of the task are at best weak and at worst nonexistent. In the case of mental practice studies only loose control over the number of practice trials is possible, for example by requiring the subject to indicate the beginning or end of each mental trial, a control which is rarely used in practice. Second, although the typical scale uses anchor points ('no image at all' to 'as bright and clear as the real thing') there can be no independent check on the assumed linearity of the scale or the consistency of judgements. The validity of these procedures thus depends primarily on the consistency of the results within some theoretical framework or model of the underlying abilities and cognitive processes. Given these severe limitations the use of objective criterion measures is attractive and investigators have recorded physiological changes in muscle activity, eye movement or brain physiology which are not only objective in the sense of being publicly observable data, but are presumed to be free of judgemental bias. The problem of compliance, however, remains since it is impossible to distinguish the subject who reports an image with no identifiable physiological change from the subject who is simply not following instructions.

The indirect methods which require subjects to perform tasks thought to require imagery processes are, of course, more robust with respect to subject compliance although it is still not possible to distinguish between compliance and capability. Imagery processes, like other cognitive processes have the status of theoretical constructs to be investigated by the usual experimental methods of cognitive psychology including the systematic manipulation of independent variables (for example the angle of rotation in mental rotation studies), the subtraction method and interference methods. The subtraction method requires the comparison of performance on two closely related versions of a task, for example simple vs two-choice reaction time. The additional time taken by the twochoice task is taken as a measure of the process of choosing. The same principle applied in brain physiology compares records of two versions of a task, for example making a fist and making an imaginary fist in order to determine what processes are necessary to both and by inference what are specific to the imagery condition. The interference method assumes a set of single function processes which can be occupied with only one task at a time. Thus if task A interferes with the performance or recall of task B then they share at least one process. These indirect methods can also relate strongly to both psychophysiological and psychoneurological investigations.

\section{INDIVIDUAL DIFFERENCES IN MOTOR IMAGERY}

Since Galton's pioneering study [36] questions about imagery have been approached from an individual differences perspective and a variety of instruments have been developed. These fall into three main categories: (1) inventory questionnaires in which subjects report the kinds of imagery they experience or use in different situations, (2) subjective rating scales, usually ratings of vividness, and (3) objective tests of performance on tasks believed to require the use of imagery. All three types are relevant to motor 
imagery particularly in assessing the role of imagery in athletic training and performance. Examples of the first are the Individual Differences Questionnaire (IDQ) [68] the Psychological Skills Inventory for Sports (PSIS) [61] and the Imagery Use Questionnaire (IUQ) [42]. The best known examples of the subjective rating scales are the Vividness of Movement Imagery Questionnaire (VMIQ) [46] and the Movement Imagery Questionnaire (MIQ) [41]. In the third category fall tests based on mental rotation such as the Mental Rotations Test (MRT) [97] and visuo-spatial working memory such as the Brooks Matrix Task [10, 20].

The IUQ is a 37-item questionnaire asking questions such as "to what extent do you use mental imagery in your training ?" and "when you use mental imagery do you see yourself from outside of your body as if you were watching yourself on a video ?" The questions cover the frequency of imagery, its relationship to training and competition and content features such as whether it is primarily visual or kinesthetic and the extent to which it includes images of success or failure. It was given to nearly 400 athletes [42] representing four different levels of expertise in six sports including 'open' skills such as football, ice hockey, soccer and squash and two 'closed' skills, gymnastics and figure skating. The results confirmed and extended previous findings [60] that the more advanced the athlete the greater the use of imagery in training, during a competition and even in bed, perhaps indicating a greater commitment to their sport. However, top level athletes were more likely to report complex imagery representing the whole of their skill and to use imagery in a more systematic way than novices. Gymnasts and figure skaters are found more likely to report 'inner view' imagery, also confirming the findings of Mahoney et al. [60] and perhaps reflecting the greater focus of their attention on kinesthetic sensations in actual performance. In a follow-up study of 348 rowers by Barr and Hall [13] the 'elite' sportsmen reported more kinesthetic imagery as did elite skiers [81]. However, Hall et al. [42] note that many athletes report the use of both internal and external views and can readily change from one to the other.

The kind of test most often reported as measuring imagery ability requires subjects to rate the vividness of images. This method was employed by Sheehan [85] in a revision of Betts' questionnaire which included questions on visual, auditory, tactile and other imagery modalities, including movement. Isaac et al. [46] developed a 24-item questionnaire specific to movement, the VMIQ, which asks respondents to rate the vividness of items such as kicking a stone or running upstairs on a five-point scale from "perfectly clear and as vivid as normal vision" to "no image at all, you only 'know' that you are thinking of the skill". The subject is requested to image each item once from an external and once from an internal viewpoint. Hall et al. [41] have produced a similar instrument in which the subject is required to perform and then image a series of meaningless actions such as "raise your right knee so that you are standing on your left leg with your right leg bent at the knee. Now lower your right leg so you are once again standing on your two feet. Perform these actions slowly". These items are then rated separately for the vividness of the subjective visual and kinesthetic experience in relation to the actual action.

Although the proponents of these tests claim both reliability and validity their predictive value remains controversial. Isaac et al. [46] report retest reliability of $r=0.76$, a figure comparable to the Vividness of Visual Imagery Questionnaire (VVIQ) with which it correlates quite well $(r=0.81)$. Hall et al. [41] report retest reliability of the MIQ of $r=0.83$. However vividness, whilst internally consistent, is a poor predictor of performance on other imagery tests and on mental practice results. Isaac et al. report it 
predicts benefits from mental practice and Marks [62] claims it predicts reminiscence effects in tracking. Hall et al. [40] report that expert performers tend to be better imagers, but most results fail to confirm a strong link between performance and vividness [22, 77]. Several studies have attempted to link imagery vividness scores to performance on spatial memory tasks. Moody [64] found subjective imagery measures did not predict memory for briefly displayed motor acts whilst Housner and Hoffman [44] found a statistically significant but not very strong effect of imagery ability on the retention of the simple linear movement. This was due to better retention of the end point location than distance and can thus be ascribed to spatial rather than motor imagery. However Walshe, Russell and Imanaka [101] failed to confirm this effect. Hall et al. [40] classified their subjects as high or low imagers on the basis of the MIQ and the learning task comprised a series of spatial patterns which subjects saw and were then required to reproduce both immmediately and then some days later. The results showed no significant effect of imagery ability on the accuracy of immediate or delayed recall.

Vividness measures appear to predict performance on other imagery tasks rather weakly or not at all. Dean and Morris [22] found that the VVIQ, which correlates quite well with the VMIQ, did not correlate with the MRT or with other spatial tests. The psychometric structure of various supposed tests of imagery currently presents a somewhat confused picture. Pavio and Harshman [72] found a main verbal and a main spatial imagery factor in their analysis of answers to their IDQ but also extracted a separate vividness factor but few of their questions relate specifically to motor imagery. In a later study Paivio and Clark [71] distinguish static from dynamic imagery. Whilst vividness is a main characteristic of static imagery controllability characterises dynamic imagery implying a motoric element in the latter. Paivio and Clark report finding male superiority in dynamic imagery tasks even suggesting that this dimension may better predict athletic performance and the ability to benefit from mental practice but much more work remains to be done before these speculations can be confirmed.

The results of factor analytic studies are inevitably influenced by the data selected for inclusion. Kosslyn et al. [54] selected a number of imagery tasks thought to represent component structures and processes in a general model of visual imagery including shortand long-term memory structures and processes such as image generation and scanning. A sample of 50 subjects was tested on 13 imagery tasks. The pattern of intercorrelations led to the conclusion that they did not tap a single imagery ability or even general cognitive ability. Three interpretable factors emerged. The first factor is taken to represent a 'picturing' or generative process, the second a 'resolution' process which is involved in image transformation tasks and the third a 'regeneration' process which maintains the image over time. Unfortunately the tasks in this study were primarily visual and, although this approach is potentially productive the results give us little guidance on the nature of motor imagery as such. We are, therefore, left with the conclusion that although subjects are willing to rate the vividness of their images of movement this bears little relation to their ability to use imagery effectively in mental rehearsal of physical tasks and probably has little to do with mental manipulation of images.

\section{MENTAL PRACTICE}

The use of imagery is commonly reported by elite athletes $[60,96]$ and is recommended by sports psychologists as part of an athlete's program of mental preparation but there is 
considerable variety in the purposes and techniques employed. For example, Orlick [67] recommends the use of imagery to "see success", that is to formulate performance goals and to motivate the attainment of those goals, to familiarise oneself with the environment, for example the quirks of a particular ski course, as a reminder of the key elements of the performance requiring attention, to facilitate warm-up and general preparedness and to perfect skills by mental practice. Most of these procedures are likely to involve motor imagery and sports psychologists recommend that imagined performances should be felt as well as visualised. However, a major part of the experimental literature concerns mental practice which Richardson [76] defined as "the symbolic rehearsal of a physical activity in the absence of any gross muscular movements", used for the purpose of acquiring or maintaining a skill, usually as a supplement or an alternative to overt physical practice.

The literature on mental practice is extensive $[26,27,32,65,69]$ and generally leads to the conclusion that various forms of imaginary practice can have measurable effects on performance. There is, however, little agreement as to the mechanism by which these effects are obtained. Paivio [69] suggests that the role of imagery in simply motivating the athlete may have been underestimated whilst Murphy [65] complains that it is difficult to draw any general conclusion from the varied techniques and paradigms which have appeared in the literature under the heading of 'mental practice'. Driskell et al. [26] in a recent meta-analytic study of some 35 carefully selected published reports reached the conclusion that mental practice has a significant effect on subsequent physical performance, although the effect is usually less powerful than that achieved by overt physical practice. Hall, Rogers and Barr [42] and Barr and Hall [13] report that elite athletes who use imagery techniques are significantly more likely to use the internal viewpoint and to experience 'feel' than are novices. Ulich [95] noted a higher level of muscular activity in subjects who benefited most from mental practice. Ungerleider and Golding [96] however report that Olympians, compared with Olympic trialists, tend to use more external perspectives but also report stronger physical sensations accompanying their imagery.

The psychoneuromuscular theory [76] is based on the hypothesis that kinesthetic feedback derived from minimal muscular activity is enough to support learning and is consistent with the view expressed by Jacobson [47] that mental activity is essentially suppressed physical activity. An alternative theory proposed by Sackett [82] and receiving some support from Feltz and Landers [32] is that mental practice is effective to the extent that motor skills usually involve some feature which can be encoded, and hence rehearsed symbolically and that it is these cognitive aspects of the skill which benefit from mental practice. Such features might include a mental plan or motor program which specifies the order and timing of responses or which enables the performer to chunk information in an efficient way. According to Driskell et al. [26] the weight of evidence supports the symbolic theory, at least to the extent that skills requiring less physical effort benefit more than those requiring greater effort and presumably entailing more muscular involvement, although positive results can be obtained with both cognitive and physical tasks. Experience moderates the effects of mental practice and Driskell et al. report an interaction between type of task and amount of experience such that whilst experienced subjects benefit equally from mental practice on cognitive and physical tasks novices benefit more from mental practice on cognitive than physical tasks. This finding is consistent with the notion that novices may be still at the 'cognitive' stage of skill acquisition [33]. 
A theory of the acquisition of serial skills developed by MacKay [58, 59] is consistent with the symbolic theory. The theory describes a hierarchy of interconnected nodes which are responsible for controlling an organised sequence of behaviour. The network ensures that task elements occur in the correct sequence and is capable of learning through practice so that the time to produce a correct sequence is reduced by repetition. At the top of the hierarchy is a node representing the whole behaviour in an abstract form whilst nodes controlling individual muscles are at the bottom of the hierarchy. In general activation spreads from the top down through the network of nodes and each node is primed when the nodes connected to it become active.

The following example illustrated by Fig. 1 was suggested to the author by Don MacKay and it represents the serial skill of tying a bow. The 'bow tying node' at the top of the hierarchy is presumed to have been triggered during the execution of some even higherlevel procedure such as getting dressed or making a parcel. Two subordinate nodes represent two partial knots each comprising a preparatory phase of grasping the free ends, making loops and crossing them and a second executive phase twisting the ends or the loops together and tightening the knot. The details are described in terms of simpler nodes referring to actions such as grasping, twisting together, making loops and pulling tight. These are mental nodes and each is primed when the superior node is activated. Only one node is active at a time and the order in which they are activated is controlled by a mutual activation/inhibition process such that when nodes to the left are activated those to their right in the diagram are inhibited, thus knot 2 cannot be executed until knot 1 becomes inactive on completion. At the lowest level are nodes representing the finger, hand and arm muscles. Primed nodes do not become active until triggered and, at least in the case of the muscle nodes, the triggering mechanism is under voluntary control. During mental practice the triggering of muscle nodes is voluntarily inhibited but the mental nodes are primed and activated in exactly the same way as happens during physical practice.

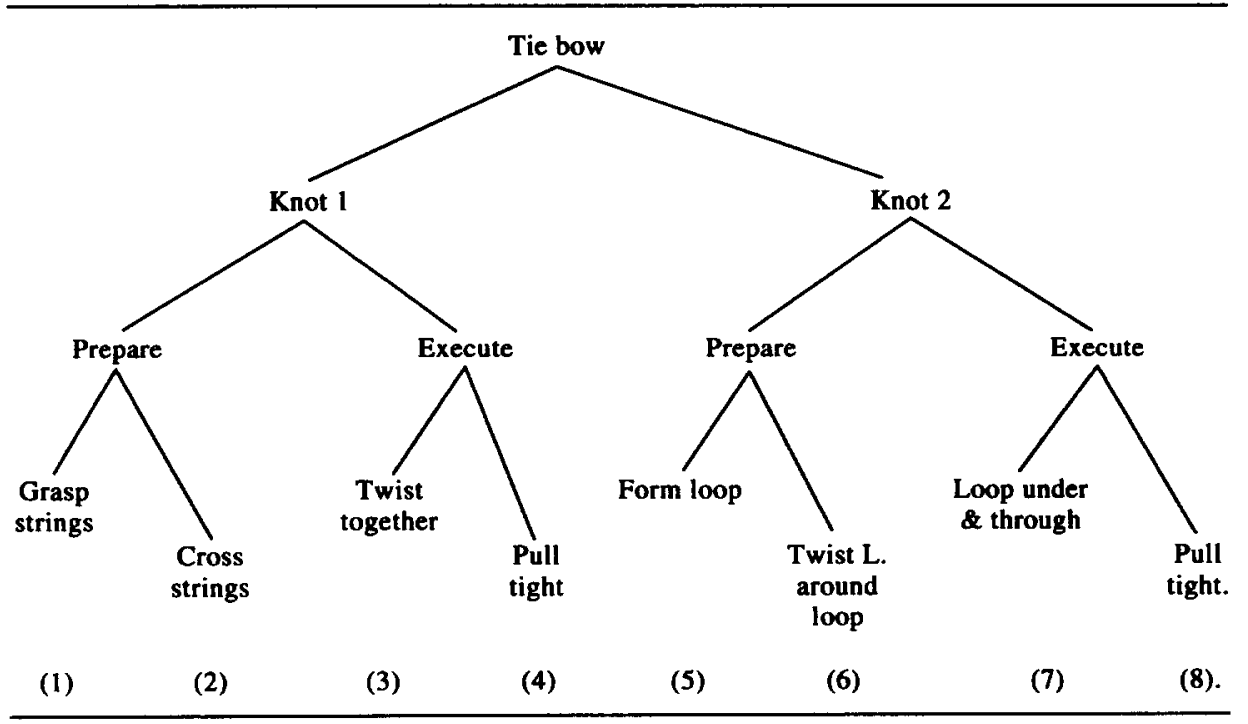

Fig. 1. A putative control hierarchy for the serial skill of tying a bow based on a suggestion by D.G. MacKay. Each node activates subordinate nodes but nodes to the right are inhibited whilst those to their left remain active. 
A simple learning rule is postulated, namely that the more frequently a node has been activated by another node the faster it will be primed. Thus when a particular sequence is practised priming rates will increase, activation will occur sooner (but only in the order determined by the triggering mechanism) and so the skill will be performed faster. The rate at which this learning process occurs will approximate the log-log linear law of practice [66]. A critical point in the theory is that whilst the same rate of learning occurs at all levels of the hierarchy the higher level connections occur less frequently, for example similar finger movements are required in tying all kinds of knots but only the sequence shown at the lowest level in the diagram produces a bow. This means that the connections between higher level nodes should benefit more from a given number of practice trials than the lower level nodes. It is further suggested that during mental practice only the mental nodes are activated and therefore serial skills should benefit at least as much from mental as from physical practice. The theory does not incorporate a feedback mechanism, although it is clear that feedback is a necessary feature of serial skills such as speech production [1]. The absence of feedback is perhaps the most puzzling feature of learning by mental practice and MacKay's theory offers a learning mechanism immune to the absence of feedback. The theory also offers a plausible explanation of the phenomena of transfer and motor equivalence insofar as high level nodes may determine a general course of action which may be executed by different sets of muscles on various occasions according to temporary or local constraints and requirements.

MacKay has illustrated the theory with a speech production task [58] in which subjects are required to practise sentences either aloud or silently, including sentences with scrambled word order and sentences with the same meaning but in a different language. Improvements in speed of production was observed under both physical and mental practice conditions but trials were always faster in the latter case. Interestingly transfer from practice in one language to production in another was found to be slightly greater after mental than after physical practice. The only known attempt at replicating these findings (albeit using different tasks) have not been entirely successful. Beladaci $[5,14]$ had subjects practise copy typing short sentences either physically or mentally with physical practice on pre- and post-tests. Some sentences were scrambled and others were presented normally, the latter material having greater pre-experimental connectivity at the higher level nodes. Subjects included both skilled typists and novices since MacKay's theory would predict that the former would benefit from greater connectivity at the muscular nodes. It was predicted that for normal material unskilled typists would benefit more than skilled typists from physical practice whilst skilled typists should benefit more from mental practice with scrambled sentences. The results showed that typing speeds improved significantly with both mental and physical practice and, unsurprisingly, that skilled typists were faster than unskilled typists and that scrambled sentences were typed more slowly than normal sentences. However, the key predictions that skilled typists should benefit more from mental than physical practice and that mental practice would be relatively more effective for scrambled than normal sentences were not confirmed. In fact the unskilled typists showed marginally more improvement with mental practice. We are forced to conclude that one of the most promising theories of mental practice effects still lacks solid empirical support.

Given the sheer variety of evidence which has been adduced for mental practice effects and the long period over which the research effort has extended one should not expect that a single theory would comprehend all the results obtained. In all probability several 
distinct mechanisms are involved. First, frequent use of imagery by athletes may well be associated with high levels of motivation to succeed and this will be associated with wellfocused attention to the key tasks and greater expenditure of effort and this could be of value in many different sports. Second, the kinds of planning process implicit in MacKay's theory still has some plausibility in view of the value which many practitioners of serial skills from slalom skiing to guitar playing place on mentally rehearsing series of rapidly executed movements. Carefully controlled experiments using the kinds of techniques used by Rosenbaum and others to study motor programs [80] could yield more satisfying evidence of the facilitation of high level motor control programs through mental rehearsal. Third, the role of imagery in more global bodily preparation for action cannot be ignored. There can be little doubt that generalised states of arousal can be brought under voluntary control through imagery but more specific processes than this may be brought into play simply by entertaining an intention. Preparation for action can be noted at the cortical level (bereitschaftpotential), in visceral responses and at the neuromuscular level in terms of preparatory postural adjustments including the facilitation or inhibition of spinal level reflexes. It may well be that these 'intentional' processes which normally precede overt action are equally activated by imagery and that the facilitation generated contributes to the efficiency of the action even when it is delayed. Such a process may well be of value in sports which rely on the highly accurate release of energy in aiming at a target.

\section{COGNITIVE PROCESSES IN MOTOR IMAGERY}

Cognitive psychology offers alternative methods for the investigation of imagery, including motor imagery by providing the subject with a task or problem which calls on imagery processes for its solution. Two variants will be discussed here, first a task in which the subject is required to provide a factual answer to a movement related question, for example "how do you tie a bow?" and the second which requires the subject to retain movement related information in short term memory by observing and then recalling a series of actions. In both cases introspective reports lead us to assume that imagery plays a significant role in task performance but the reports themselves are not treated as data for the purposes of testing hypotheses concerning the functional properties of imagery predicted on the basis of a theoretical model. Two such models will be discussed, one by Baddeley and his co-workers $[10-12,57]$ and a less well-known model by the present author [2]. The Baddeley model was developed to account for the phenomena of shortterm or working memory which is characterised by the maintenance of newly presented material in current consciousness for almost immediate recall. The Annett model was developed as a means of coordinating various hypotheses to do with the role of cognitive processes, especially verbal instruction and imitation, in motor skill acquisition. The two models have a number of common features including a mechanism for motor imagery.

When a subject is asked to "tell me in as much detail as you can how to take two ends of string and tie them together to make a bow" the characteristic first response is to show some sign of mild embarrassment and avert the eyes. There is often a short hesitation and the subject may ask "am I allowed move my hands?". Gestures often accompany the verbal explanation which is typically halting, sometimes with repetitions and corrections. Whereas most people can tie a bow without undue haste in about $10 \mathrm{sec}$, explanations normally take about three times as long and by no means all of this extra time is taken up in speech. Subjects invariably report that they rely on imagery to support the explanation 
and that hesitations and failures are due to the loss of the image. The imagery is reported to be fragmentary, usually in the form of short scenes like still snapshots or short sections of 'movie' which represent particular stages of the process. The stages most often referred to in the verbal protocol refer, perhaps not surprisingly, to essential sub-goals such as crossing the two ends, forming a loop or tightening the knot. It is clear that what is being recalled is information which helps to specify these sub-goals in the form of images which can be used to cue the subsequent move. Hesitations typically occur between these stages the longest typically being after the first half knot has been completed and subjects report having to make a special effort to generate an image of the next stage. For this task subjects mostly report internal view imagery in which they are themselves active rather than just a passive observer although some other tasks such as "tell me how you get on a bicycle" or "tell me how you do a somersault" are more likely to yield reports of an external view.

A special feature of this task is that imagery appears to play an essential part in the link between the implicit or procedural knowledge of bow tying and the explicit or declarative knowledge required to give a verbal explanation. I have referred to this as the actionlanguage bridge and suggest that it can operate in either direction, that is in generating verbal instructions - explaining how to do something-and in following instructions. These functions are fundamental to the early stages of skill acquisition where demonstrations facilitate learning but have a much wider import in terms of understanding from verbal accounts what is going on in the world and of effecting real world changes vicariously by issuing orders. The model postulates that, this crucial link apart, the verbal and non-verbal systems operate independently. Evidence in support of this postulate comes from studies which demonstrate the independence of declarative and procedural memory. Corkin [19] showed that the much studied amnesic H.M. had near normal capacity to acquire a tracking skill, and evidence that the action-language bridge is correctly conceived as a link which can be broken comes from studies of apraxia, especially those cases attributed to callosal damage or 'disconnection syndrome' [38]. Added support for the verbal-motor independence come from the adoption by Paivio of the dual coding hypothesis [68, 70] and Baddeley's two-part working memory model to be discussed shortly. However, it is the perception-action link which is of central importance to the present discussion of motor imagery.

The basic model proposes independent perceptual-motor and verbal systems. Each comprises a receptive-representational sub-system and a motor executive sub-system. The perceptual-motor and verbal systems are linked at the representational level and this link is referred to as the action-language bridge. For example, in receiving and following instructions a route is established between the auditory-verbal representational system across the action-language bridge to the motor representational system and thence to the motor executive system. By contrast when issuing instructions (or giving an explanation) the perceptual-motor system is activated and information is passed across the actionlanguage bridge to the verbal representational system and thence to executive speech output. Processes such as verbal shadowing and mimicry are represented by direct links in which externally derived information activates the receptive-representational sub-systems which then activate the executive sub-systems leading to motor output. Imagery processes, either auditory-verbal or perceptual-motor are presumed to be due to activation of the representational systems but these are intimately linked to the corresponding executive systems. The nature of this link is of central importance. James [48] reported an extreme 
example of motor involvement in imagery, a Professor Stricker of Vienna, a self described 'motile' whose recollections of both his own movements and those of others were invariably accompanied by distinct muscular feelings in those parts of his body which would normally be used in effecting the movement. In thinking of a soldier marching it was as if he were helping the image to march by marching himself. If he suppressed this sympathetic feeling and concentrated attention on the imagined soldier the latter became as if it were paralysed. The same Professor Stricker also claimed that verbal images become indistinct if the tongue and mouth are held still, for instance by gripping a pencil between the teeth.

The observation that involuntary movements frequently occur when the subject is imagining a series of actions such as how to tie a bow [3,6] seems to lend further support to the view that motor imagery involves activation of the motor output system. However, gestures which typically accompany tasks requiring action imagery do not exactly replicate, even in miniature the exact form of the movements which comprise the physical task. For example, in trying to imagine how the loops of a bow are intertwined subjects are as likely to use a forefinger to represent the string itself as to reproduce the finger movements actually made when manipulating the string. Figure 2(a) shows a subject actually making a loop whilst Fig. 2(b) shows the gesture used by the same subject explaining how to make a loop. The imaginary bow usually employs gestures which are of greater amplitude and contain less fine detail. For example the hands indicate the shape and direction of the movement but fine finger movements are typically not seen. If movement of the hands is restricted some other part of the body, for example the head, may be used to indicate the spatial direction of the imagined movement which certainly suggests that an approximate or generalised motor image is being brought into play rather than a detailed record of muscular reactions. An alternative view of the role of involuntary gestures accompanying verbal explanations is that they are more closely related to speech output than to the actions represented in imagery and that their role is emphatic rather than being directly representative of motor imagery. McNeil [63] distinguishes 'iconic' gestures which take the form of the idea being expressed and 'beat' gestures which are simple in form and are synchronised with speech stresses. Observations taken from video recordings of subjects explaining how to tie a bow [6] revealed that there was a fairly close correspondence between the expressed ideas (grasp the strings, twist them together, etc.) and the gestures and they mostly fall into McNeil's iconic category. The temporal relationship between the onset of the verb and the onset of hand gestures to which it relates was that, on average actions followed words by about $90 \mathrm{msec}$ except in cases where there is a long hesitation and the subject appears to be struggling to generate an image when the gesture typically anticipates the verb. It is as if the executive system needs to be activated to a higher level in order to generate a clear image. When subjects are asked to demonstrate bow tying with an accompanying commentary the hand movements typically precede the relevant speech episode by about $100 \mathrm{msec}$ suggesting that procedural memory is being used as the primary source for verbal expression. Although this evidence is still sketchy it does suggest that whilst the timing of the gestures may be linked to speech output their form does relate to some presumed motor schema of the kind hypothesised by Schmidt [83] which also acts as a source for the subjectively experienced imagery.

A similar idea was proposed by Whiting and den Brinker [99] who referred to a central representation or image of the act as something more abstract than a set of implicit muscle movements but as a representation of the gross form of the action, including spatio- 

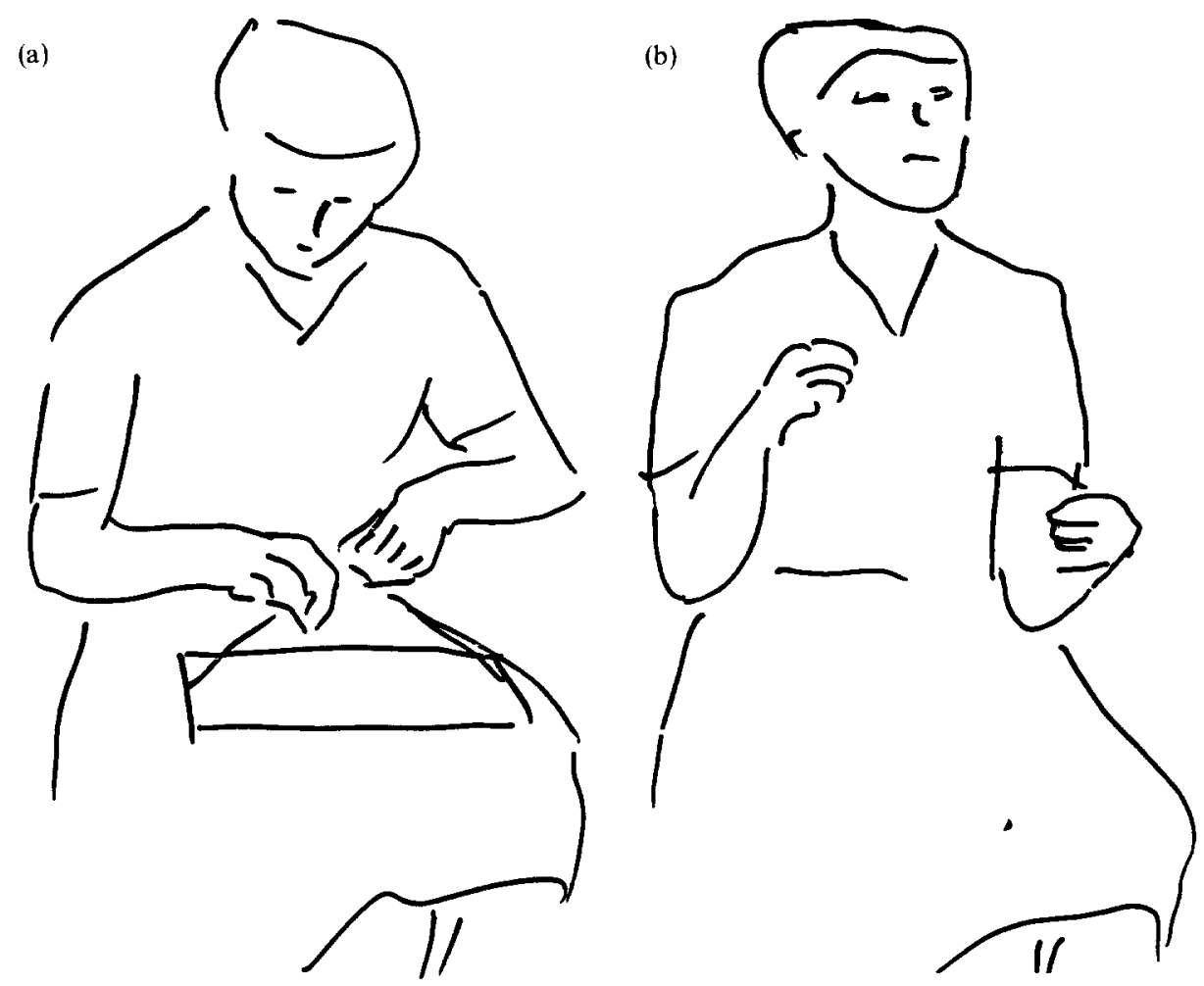

Fig. 2. Tracings of still frame from a video recording of a subject tying a bow using two pieces of string attached to a board (a); the same subject gesturing whilst giving a verbal explanation of how to tie a bow (b). In both cases the subject has reached stage 4 as illustrated in Fig. 1.

temporal relativities unconstrained by specific contextual data following Shaffer's suggestion [84] of a hierarchy of abstract representations which are used in a flexible way to construct specific musical performances according to the intended style or mood. Yet another suggestion [2] is that motor memory includes a store of action prototypes which are used in both perceiving and producing action patterns. These prototypes are often used by sports coaches to convey complex information about movement and posture. For example one coach describes the posture adopted for putting as like sitting on a bar stool whilst a squash coach describes the stance for receiving service as "as if you are on the war-path waving a tomahawk". Stanislavski the founder of the 'method' school of acting frequently stressed the importance of inner representations in expressive movement. "Our spinal column ... is like a spiral spring ... and needs to be firmly set on its base. It must be, as it were, screwed into place. If a person feels this screw is strong the upper part of his torso has a support, a centre of gravity, stability and straightness. But if on the contrary he feels as if the screw is undone, his spinal column, and hence his whole torso loses its stability ..." $[92$, pp. 43,44$]$ and again, "... when we are on the stage our chief concern should be to reflect at all times in our own inner vision the things akin to those our character would have in his ... once we have established inside ourselves this easily accessible sequence of images our task of keeping on the right lines of the subtext is greatly lightened ..." [92, pp. 124, 125]. 
These qualitative observations find some support in controlled experimental studies of human movement perception and imitation. Johannson [51] has demonstrated that meaningful features of human movement are detectable from minimal cues. In these wellknown experiments stimuli are prepared by filming a dark-suited actor in low illumination with small lights attached to the main body segments. Whilst the actor remains still only an apparently random pattern of light points is seen but virtually as soon as the actor moves the light stimuli resolve into meaningful action and fine judgments can be made about what the actor is doing, what load is being carried and even the sex of the actor can be guessed better than chance. Whiting and his associates [98] have shown that subjects learning a simulated ski task by observing a model appear to be able to pick up certain qualitative features of the model's performance and incorporate these into their own responses in a way which is consistent with the existence of a set of specifications of perceptual-motor movement parameters which are functional in both perceptual and motor terms. Studies of the role of movement in short-term memory shed further light on the joint perceptual and motor functions of imagery.

\section{Visuo-spatial working memory}

The working memory paradigm requires the subject to memorise material presented rather than to generate images from long-term memory. Imagery is nonetheless involved, either explicitly as in the Brooks task where the subject is instructed to try to remember a series of spatial locations by mentally placing numbers in the cells of an imagined matrix, or implicitly in other experiments on memory for visuo-spatial information. The model developed by Baddeley and his associates $[10-12,57]$ postulates two sub-systems, one verbal and one visuo-spatial. As in the Action-Language-Imagination model [2] each subsystem comprises a receptive and an executive component. The receptive component comprises a sensory buffer in which material is held in temporary store in a form fairly close to its sensory origins. Thus for auditory material held in short-term memory errors are more frequently attributable to acoustic confusion rather than semantic confusion. The sensory buffer holds material for short durations only. Not only can new material replace the old but the undisturbed trace decays rapidly unless refreshed. In the case of verbal working memory the words or numbers are maintained in conscious awareness and available for retrieval by means of an articulatory loop, that is the material is spoken subvocally and so re-enters a sensory buffer. This model of verbal working memory has extensive empirical support [10] and some of the most compelling comes from experiments demonstrating the effects of articulatory suppression. The underlying process model postulates dedicated components or resources which can only be used for one task at a time such that another verbal task, even simply repeating "bla, bla, bla ..." will interfere with the sub-vocal repetition of the to-be-remembered material, be it a set of digits or some other stimulus set for which this articulatory coding is esssential. It is argued that the 'inner ear' or short-term acoustic store, is maintained by an 'inner voice' involving some elements of the normal speech production mechanisms. The major question then is whether visual working memory, the visual-spatial sketchpad or VSSP, can be regarded as an 'inner eye' and whether information in this short-term store or visual buffer can be maintained by an 'inner scribe' which effectively reads the material from and then re-writes the material to the buffer. This is the solution offered by Reisberg and Logie [75] and it clearly implicates motor production processes in the retention of visuo-spatial material in 
working memory. As with articulatory suppression tests of the theory rely heavily on the postulate of single task resources and dual task interference experiments.

The problem which immediately comes to mind is that whilst an 'inner voice' may generate inputs very similar to the original material the 'inner scribe' may not be able to generate all the features of an original stimulus set using a single motor resource-painting pictures is a different kind of process to repeating words. Reisberg and Logie suggest that a distinction should be made between visual features such as the colour or shape of an object and its spatial location and that perhaps there might be distinct visual and spatial components to the VSSP. If this is the case then an interpolated spatial task would intefere with the spatial aspect of working memory whilst leaving the purely visual aspects untouched. Overt movements, especially when they are directed towards objects in the environment, typically provide a spatial task and will interfere with the retention of material in the VSSP. Before following up this crucial point let us first review some of the key features of motor short-term memory.

A basic motor memory task first used by Thorndike [94] requires the subject to learn and retain a simple unidimensional movement of a specified extent (for a review see [1], pp. 38-45). The task may be learned either by trial-and-error with verbal feedback or by moving from a fixed origin to a mechanical stop for any given number of trials and is then reproduced on one or more occasions. The error of reproduction, measured as linear or angular extent has been shown to depend on a number of factors including the amount and type of practice and the effects of interpolated tasks. In the context of the present discussion there are two important findings. First, the end location of the movement is recalled rather better than the absolute extent [55] and second, another movement of the same kind interpolated between learning and recall produces a systematic bias towards the extent of the interpolated movement such that a shorter movement will lead to an underestimate and a longer movement to an over estimate of the orginal extent [8, 73]. For these movements at least the essential characteristic retained in short-term memory is spatial locus of the end point. In these experiments subjects were not required to generate images or to report on them but there can be little doubt that their responses were guided by images retained in the VSSP since imaginary movements have been shown by Johnson [52] to have equivalent effects to overt physical movements on the contents of short-term memory. Using the linear positioning task, learned by moving from a fixed point to a mechanical stop for 10 trials subjects were asked to reproduce the same movement after a short interval during which some subjects simply rested, others carried out an articulatory suppression task, counting backwards in threes, and yet other groups were required to make movements which were either half or twice the orginal and yet other subjects were asked to imagine movements of half or twice the original extent. The resting subjects and the articulatory suppression subjects showed no bias in recall but those subjects who made either shorter or longer interpolated movements exhibited significant bias towards the interpolated length in their reproductions. The striking finding was that the bias induced by imaginary movement was indistinguishable in both direction and extent from that produced by overt movement. Viewed as a mental practice experiment this result is one of the strongest in the literature in showing complete equivalence between real and imagined movement.

It was suggested above that in these positioning experiments the essential memory component is spatial location rather than a record of either muscular sensations or of intended motor output or efference copy. This view is confirmed by further experiments 
[52] in which different kinds of secondary task were applied to the retention interval when the subject was also required to imagine making movements half or twice as long as the tobe-remembered movement. In one condition subjects were required to tap up and down on a single spot in time with a metronome with the same hand which they were imagining moving back and forth between the start and finishing point of the original movement. In another condition they were required to observe two lights flashing on and off at different locations and in another condition they listened to two 'beeps' of an electronic metronome located to the left and right. The results showed that only the secondary tasks containing a spatial element interfered with the imagery-induced bias confirming that whatever the subjective nature of the imaginary movements it was the spatial rather than motoric component which was producing these effects.

More complex tasks presumably involve more complex processes. Smyth and her associates have demonstrated limitations in short term memory for actions which has implications for our understanding of motor imagery. Smyth et al. [88] compared memory span for words, patterned movements of the head trunk and limbs and spatial positions, pointing at blocks in an array in a specified sequence. The normal span of about five items for words and about four items each for spatial locations was affected by a secondary task carried out during the presentation of the stimulus items. Concurrent verbal articulation (counting 1-5 repeatedly and rapidly) reduced the span for visually presented words and movements by about one item. Tapping sequentially on the head, shoulders and hips, however, reduced the span for movements by about half an item but did not affect the span for words or the span for spatial locations. In a further set of experiments [89, 90] a spatial tapping task was found to interfere with memory for spatial locations but not with memory for hand configurations whilst squeezing a flexible tube interfered with the retention of hand configurations but not with spatial locations.

Other studies of the visuo-spatial sketchpad, principally using the Brooks Matrix task have shown interference with the generation and retention of images of spatial locations due to concurrent movement. In the Brooks task the subject is asked to visualise a 4 by 4 matrix of empty squares and then to imagine the number 1 placed in square one down and one to the right of the top left-hand corner. The locations of all nine numerals are indicated verbally with reference to the starting square by telling the subject "in the next square to the right put a 2 , in the next square down put a 3 ...", etc. The typical finding in these experiments is that concurrent movement tasks such as tracking and tapping on spatially distributed targets interfere with retention, significantly reducing the number of items correctly retained. Interference has also been reported with voluntary, but not involuntary, eye movements. With some possible exceptions interference seems to be confined to the encoding phase when the to-be-remembered items are being presented rather than the retention phase. This distinction is important since it implies that concurrent movement does not disrupt the refresh mechanism of the VSSP in quite the same way that a concurrent verbal task appears to disrupt the articulatory (or phonlogical) loop.

The analogy between a refresh mechanism for the VSSP and the articulatory loop in verbal short term memory proposed by Reisberg and Logie [75] turns out, when closely examined, to have only rather indirect support and some serious theoretical difficulties. The first is that phonological coding is time-dependent in a way in which visuo-spatial coding is not just as visual identity and spatial location can be preserved in a stopped frame of a video recording but acoustic information can only be available for retrieval by 
replaying the tape. The articulatory loop is therefore a necessity for phonological coding but a parallel visuo-motor loop may not be for the retention of visuo-spatial material. Another point of comparison lies in the nature of the perceptuo-motor coupling of phonological and spatial data.

Speech production is close-coupled in that it is heavily dependent on intact auditory feedback, moreover self-generated speech closely resembles other-generated speech. The coupling between spatial perception and movement does not appear so close unless one fully accepts the Hebbian hypothesis that eye movement is fundamental to shape recognition. Perceptually identical circles and triangles can be produced by an almost infinitely varied combinations of motor activity - the motor constancy phenomenon-but the production of recognisable speech is much more highly constrained. However, the echoic and imitative nature of speech perception and production may well have a parallel in the ability to imitate bodily and facial gestures as noted by Annett [2]. What is now beginning to emerge is the possibility that the VSSP may comprise not just one or even two components but may have at least three. The first is concerned with the storage of features which identify objects and are not time-dependent, for example their shape and colour, whilst the second type preserves spatial information (both relative and absolute) which may be needed in order to approach or avoid the object. The third type is specific to the encoding of action information, especially the kind of information needed to interpret and to imitate the perceived actions of others. Since it is only the latter which requires the encoding of time-dependent information it is more likely that an action-perception feedback loop would be advantageous to maintaining information in working memory.

Critical evidence for the existence of a refresh mechanism comes from interference effects of secondary tasks presented during the retention interval. In many of the earlier studies $[11,12]$ secondary tasks were presented during stimulus presentation (encoding) as well as the retention or maintenance interval. A series of recent experiments by Smyth and Scholey [91] explored the effects on memory span for spatial locations of various visual, auditory, verbal and motor tasks when presented during the retention interval. The primary task requires the subject to watch a series of three to seven square patches appear one at a time at 1.5 -sec intervals in any of up to nine different locations on a computer screen. After a retention interval of $12.5 \mathrm{sec}$ these locations are recalled by pointing at them in the order of their appearance. A memory span paradigm was used and for an unfilled retention interval a span of just over five items was established. A word repetition task administered during the retention interval did not significantly reduce the span but pointing at a different set of targets did give prima facie evidence of motoric interference during the putative rehearsal process. Further experiments, however, showed that the interference was spatial rather than motoric. If spatial targets presented during the retention interval have to be responded to interference occurs, but it makes no difference whether the response is pointing to a target on the left or right or simply saying "left" or "right". The interference due to responding seems to be highly specific to the task. For example, listening to tones from spatially separated sources interferes with span just as it did with the interpolated movement imagery effect in Johnson's experiments [52] and overt pointing responses add to the interference. However, repeating words which originate from spatially separated sources does not reduce span so we may conclude that it is not simply motor activity per se but movement in relation to a spatial target which is crucial. This finding reinforces the idea that the source of interference lies in over-writing of a representation of the space rather than in the generation of voluntary action. It would 
appear that movement as such is secondary to attending selectively to spatial targets which precedes the movement itself and is essential to the execution of an accurate motor plan.

\section{PHYSICAL CORRELATES OF MOTOR IMAGERY}

The functional equivalence hypothesis invites the question of where the processes subserving motor imagery may be located in the brain. The hypothesis predicts that many, but not all, those parts of the brain involved in overt movement should be active during imaginary movement. Jeannerod [50] suggests that motor imagery can be characterised as preparation for action and so one would expect to find activity during imagery in those parts of the forebrain believed to be concerned with action planning whilst other parts lower down the presumed command hierarchy which are active during overt movement, the primary motor cortex for example, might be less active during imaginary than during overt movement. However, since many actions are directed towards objects in space and the conscious content of motor imagery often includes visuo-spatial representations one might also expect occipito-parietal areas of the cortex and perhaps also the cerebellum to be active during motor imagery. This approach to the localisation of imagery processes, which is much encouraged by recent developments in brain scanning techniques is, however, not without difficulties. First, motor control is highly distributed throughout the CNS and the spinal cord and so most areas will have some part to play in any moderately complex perceptual-motor activity. Furthermore observable actions sometimes depend rather indirectly on specific neural activity. Highly evolved animals have acquired a number of relatively distinct motor systems [32] which may operate independently, or as is more usual, collaboratively. For example the simple action of walking towards a target object depends on the integrity of a number of distinct neural systems interacting with the mechanical properties of the body within the gravitational field and with each other. Walking may be viewed as a kind of controlled falling forward in which the oscillation of the legs is synchronised with adjustments of the postural muscles of the head and trunk in such a way as to take advantage of the force of gravity acting on the limbs. Very little conscious attention is normally devoted to these mechanisms but at the same time rate and direction of walking are controlled by visual cues, the seen objective and the systematic expansion or contraction of objects in the visual field [56]. As Jeannerod [50] points out we must not assume that all aspects of observable action have an identifiable representational basis. It is also clear that a great deal of motor activity is normally unconscious and automatic and is therefore unlikely to feature in motor imagery which, is by definition, conscious.

A number of recent studies have tackled the localisation question through brain scanning and imaging methods $[23,24,34,45,74,79]$ and have concluded that movement imagery involves widely distributed cerebral structures, but particularly those areas in the frontal lobes believed to support action planning and other areas, especially the parietal lobes, believed to support spatial representations in working memory. They have usually shown reduced activity, as measured by regional blood flow, in the primary motor cortex during imaginary as compared with overt movement [45, 79]. EEG studies using alpha suppression as the index of cortical processing [16, 21, 16, 100] have on the whole confirmed the impression that both motor planning and spatial awareness are involved in motor imagery. Scanning technologies, however, present a number of problems. First, whilst the spatial resolution may be good, at least in the case of positron emission tomography (PET) and magnetic resonance imaging (MRI), temporal resolution still 
leaves a lot to be desired given that most discrete actions are executed in something less than $1 \mathrm{sec}$. In order to obtain a record the actions the subject is required to perform are usually simple repetitive hand movements which are not necessarily representative of normal free-flowing activity. Only event-related potential (ERP) techniques can match the temporal evolution of a single motor act from preparation through execution to terminal control, but the number of such studies is as yet small [36] and they also require 30-40 repetitions to acquire an adequate waveform. A further serious limitation is that the recording equipment restricts the range of actions which can be studied since imaging techniques typically rely on a subtraction methodology in which data acquired during imagery is compared with data from an overt movement condition. For this reason most published work relates only to simple repetitive hand movement.

The subtraction method is less constraining when comparing visual inspection tasks with visual imagery and a small number of studies have looked for evidence of motor involvement in mental rotation. Williams et al. [100] found evidence of alpha suppression in both pre-motor and parietal regions during the MRT [97], whilst Farah and Perronet [31] using an ERP paradigm found increased negativity between 400 and $800 \mathrm{msec}$ after stimulus presentation to an extent dependent on the degree of rotation required. This latter result finds confirmation in a study of single cell recording by Georgopoulos et al. [37] in rhesus monkey. The animal was required to mentally rotate a pattern in order to make a rewarded response. The directional tendency of the neuronal population in the motor cortex was found to respond systematically during reaction time.

The study of imagery deficits resulting from brain pathology provides an alternative approach to localisation questions. A number of studies have reported imagery deficits mainly related to lesions in the right parietal areas $[9,28-30,43,53,78]$. None of these report specific deficits in movement imagery but visuo-spatial imagery could affect some kinds of imaginary actions. For example Hanley et al. [43] did find an impairment in mental rotation as well as other spatial imagery tasks in a patient with a right hemisphere aneurysm. Farah et al. [30] report a case of bilateral lesion where the difficulty appeared to be mainly in generating images. With regard to lesions in sub-cortical structures Annett and Smith [7] showed that patients with Parkinson's disease whose ability to tie a bow was severely affected were able to produce normal responses in the imaginary bow tying task. The dissociation of perceptual from executive function in Parkinson's disease was confirmed by Stelmach et al. [93] and these results therefore suggest that movement imagery is more likely to involve planning and preparation than executive processes. Especially relevant to the question of where motor imagery lies in the putative inputoutput chain is a report by di Pellegrino et al. [25] of a group of neurons in the pre-motor cortex (area 6) in Macaca nemestrina which fire prior to and during specific directed hand movements and also when the animal observes another individual making the same movement. This finding may represent the operation of a mechanism which could support action prototypes which participate in both the perception and production of habitual movement patterns as proposed by Annett [2].

\section{SUMMARY AND CONCLUSIONS}

How then can we characterise motor imagery? Is it genuinely motoric, some kind of incomplete action, or is it purely perceptual, a combination of visual and kinesthetic imagery? The evidence suggests that a number of distinct processes may be involved in 
different episodes of motor imagery, and so a componential model of the type proposed by Kosslyn [53,54] is required. If we take explaining how to tie a bow as a typical naturalistic example of motor imagery the following kinds of processes seem to be involved. First the requirement to provide an explanation challenges long-term memory and so the immediate response is a shift of attention from external to internal data. This step is often signalled externally by eye movement, a shift of gaze away from the former focus of attention. However in this case there is no readily available verbally encoded or declarative account of bow tying to retrieve and place in the sensory buffer from whence it can be converted into speech output. Bow tying, like many other motor skills, is encoded in a separate procedural memory. The process of searching procedural memory means accessing appropriate generalised motor programs or prototypes together with their sensory concomitants, in this case visual images of string and parcels or shoes and laces. Single images however vivid are not enough to reconstruct the full procedure but continued activation of the prototype will produce new images of subsequent steps which are then available in the sensory buffer and the verbal system. Even a relatively simple procedure like bow tying cannot be entertained as a whole in the sensory buffer but must be constructed step by step and this involves generating a series of moves and confirming from the evidence in the visual buffer that the imaged move will contribute correctly to the overall goal. The process of retrieving action prototypes from long term memory involves activating them but the actual motor output is not exactly like that produced in the course of fully overt action because in this case there is no requirement to incorporate new sensory data such as the actual location, mass, resistance and other physical properties of the items to be manipulated. Overt movement, even on a small scale, may not even be necessary if the requirements of the task can be met without it. Open skills depend primarily on using spatial information so simple skills such as pointing to the remembered location of an object will depend less on the activation of a detailed motor program but more on the retention of a representation of space. There is plenty of evidence to suggest that movement per se does not disrupt spatial memory provided it does not entail use of the spatial array. However, shifts of attention can be disruptive when they entail a new spatial reference. Closed skills on the other hand are characterised more by changes in posture and the relative positions of body parts. Imagining such skills is more likely to produce both discernible movement and subjective reports of an internal view with kinesthetic imagery.

The evidence emerging from physiological and neurological investigations lends support to the conception of a highly distributed system supporting motor imagery. Motor imagery does not appear to depend on the integrity of executive motor systems such as the primary motor cortex and the basal ganglia but both physiological and neurological evidence point to the involvement of the parietal and temporal areas and parts of the frontal cortex. There is evidence of groups of neurones in the frontal lobes which encode both perception and production of meaningful actions and parts of the temporal lobe which code motion patterns, including human gait, whilst spatial location which is important to open skills seems to depend on the parietal lobes. Improved techniques for direct recording from active cells and new brain imaging techniques provide a promising way forward but neurological studies could also throw light on the processes underlying motor imagery.

The functional theory of motor imagery outlined above offers no detailed explanation of the benefits which some athletes claim for mental rehearsal. Motor imagery however may 
be seen as embedded in an associative network with connections to other systems through which cognitive events may have an effect on performance through controlling states of arousal or by focusing attention or by priming different neuro-muscular systems for action. These somatic functions do not, of course, rule out possible cognitive effects which could include both consolidation of motor memories and also the reorganisation of action plans. But these are distinct hypotheses which must be investigated in specific experimental designs. We should not expect one theory or a single experimental paradigm to provide a general answer to all these problems.

\section{REFERENCES}

1. Annett, J. Feedback and Human Behaviour. Penguin Books, Harmondsworth, 1969.

2. Annett, J. Action, language and imagination. In Psychology of Sport and Motor Behavior: Research and Practice, L. Wankel and R. B. Wilberg (Editors), pp.271-282. University of Alberta, Edmonton, 1982.

3. Annett, J. On knowing how to do things. In Generation and Modulation of Action Patterns. H. Heuer and Fromm (Editors), pp. 187-200. Springer, Berlin, 1986.

4. Annett, J. Motor learning and retention. In Practical Aspects of Memory: Current Research and Issues, M. M. Gruneborg, P. E. Morris and R. N. Sykes (Editors), Vol. 2, pp. 434 440, Chichester: John Wiley \& Sons, 1988

5. Annett, J. Imagery and skill acquisition. In Cognitive and Neuropsychological Approaches to Mental Imagery, M. Denis, J. Engelkamp and J. T. E. Richardson (Editors), pp. 259-268. Martinus Nijhof, Dordrecht, 1988

6. Annett, J. Relations between verbal and gestural explanations. In Cerebral Control of Speech and Limb Movements, G. R. Hammond (Editor), pp. 327-346. North Holland, Amsterdam, 1990

7. Annett, J. and Smith, R. J. Motor imagery in Parkinson's Disease. In Pre-proceedings of the 2nd European Workshop on Imagery and Cognition, C. Cornoldi (Editor), pp. 373-388. University of Padua, 1988.

8. Adams, J. A. and Dkjkstra, S. Short term memory for motor responses. J. exp. Psychol. 71, 314-318, 1966.

9. Anderson, R. A. Neurobiological basis of spatial cognition. In Spatial Cognition: Brain Bases and Development, J. Stiles-Davis, M. Kritchevsky and U. Bellugi, pp. 57-80. Lawrence Erlbaum, Hillsdale, New Jersey, 1988

10. Baddeley, A. Working Memory. Clarendon Press, Oxford, 1986.

11. Baddeley, A and Hitch, G. Working memory. In Recent Advances in Learning and Motivation, Vol. VIII, G. Bower (Editor), pp. 47-90. Academic Press, New York, 1974.

12. Baddeley, A. and Lieberman, K. Spatial working memory. In Attention and Performance VIII, R. Nickerson (Editor), pp. 521-539. Laurence Erlbaum, Hillsdale, New Jersey, 1980.

13. Barr, K. and Hall, C. The use of imagery by rowers. Int. J. Sport Psychol. 23, 243-261, 1992

14. Beladaci, A. Unpublished Master's Thesis, University of Warwick, 1989

15. Betts, G. H. The Distribution and Functions of Mental Imagery. Teachers College, Columbia University, New York, 1909.

16. Beyer, L., Weiss, T., Hansen, E., Wolf, A. and Seidel, A. Dynamics of control of nervous activation during motor imagination. Int. J. Psychophysiol. 9, 75-80, 1990.

17. Breitling, D., Guenther, W. and Rundot, P. Motor responses measured by brain electrical activity mapping. Behav Neurosci. 100, 104-116, 1986.

18. Cooper, L. A. and Shepard, R. N. Chronometric studies of thee rotation of mental images. In Visual Information Processing, W. G Chase (Editor). Academic Press, New York, 1973.

19. Corkin, S. Acquisition of a motor skill after bilateral medial temporal lobe excision. Neuropsychologia 6 , 255-265, 1968.

20. Brooks, L. Spatial and verbal components of the act of recall. Can. J. Psychol. 22, 349-368, 1968.

21. Davidson, R. J. and Schwartz, G. E. Brain mechanisms subserving self-generated imagery: Electrophysiological specificity and patterning. Psychophysiology 14, 598-602, 1977.

22. Dean, G. and Morris, P. E. Imagery and spatial ability: When introspectice reports predict performance. In Mental Images in Human Cognition, R. Logie and M. Denis (Editors), pp. 331-347. Elsevier, Amsterdem, 1991.

23. Decety, J. and Ingvar, D. H. Brain structures participating in mental simulation of motor behavior: A neurophysiological interpretation. Acta Psychol. 73, 13-34, 1990.

24. Decety, J., Perani, D., Jeannerod, M., Bettinardi, V., Tadary, B., Woods, R., Mazziotta, J. C. and Fazio, F. Mapping motor representations with positron emission tomography. Nature 371, 600-602, 1994. 
25. Di Pellegrino, G., Fadiga, L., Fogassi, L., Gallese, V. and Rizzolatti, G. Understanding motor events. Exp. Brain Res. 91, 176-180, 1992.

26. Driskell, J. E., Copper, C. and Moran, A. Does mental practice enhance performance? J. appl. Psychol. 79, 481-492, 1994.

27. Druckman, D. and Swets, J. Enhancing Human Performance. National Academy Press, Washington, D.C., 1988

28. Erlichman, H. and Barrett, J. Right hemisphere specialisation for mental imagery: A review of the evidence. Brain Cognit. 2, 55-76, 1983.

29. Farah, M. J. Is visual imagery really visual? Overlooked evidence from neuropsychology. Psychol. Rev. 95, 307-317, 1988.

30. Farah, M. J., Hammond, K. M., Levine, D. N. and Calvantio, R. Visual and spatial mental imagery: Dissociable systems of representation. Cognit. Psychol. 20, 439-462, 1988.

31. Farah, M. J. and Peronnet, F. Event-related potentials in the study of mental imagery. J. Psychophysiol. 3, 99-109, 1989.

32. Feltz, D. L. and Landers, D. M. The effects of mental practice on motor skill learning and performance: A meta-analysis. J. Sport Psychol. 5, 25-57, 1983.

33. Fitts, P. M. Perceptual-motor skill learning. In Categories of Human Learning, A. W. Melton (Editor), pp. 243-285. Academic Press, New York, 1964.

34. Fox, P. T., Pardoe, J. V., Petersen, S. E. and Raichle, M. E. Supplementary motor cortex and pre-motor responses to actual and imagined hand movements with positron emission tomography. Neurosci. Abstr. 1433, 1987.

35. Gallistel, C. R. The Organisation of Action: A New Synthesis. Laurence Erlbaum, Hillsdale, New Jersey, 1980.

36. Galton, F. Inquiries into Human Faculty and its Development. Macmillan, London, 1883.

37. Georgopoulos, A. P., Lurito, J. T., Petrides, M., Schwart, A. B. and Massey, J. T. Mental rotation of the population neuronal vector. Science 243, 234-236, 1989.

38. Geschwind, N. and Kaplan, E. A human cerebral disconnection syndrome. Neurology 12, 675-685, 1962.

39. Gross, C. G., Desimone, T., Albright, T. D. and Schwarts, E. L. Inferior temporal cortex as a visual integration area. In Cortical Integration, F. Reinoso-Suarez and A. Ajmone-Marson (Editors), pp. 291-315. Raven Press, New York, 1984.

40. Hall, C., Buckolz, E. and Fishburne, G. Searching for a relationship between imagery ability and memory of movements. J. Hum. Move. Stud. 17, 89-100, 1989.

41. Hall, C., Pongrac, J. and Buckolze, E. The measurement of imagery ability. Hum. Move. Sci. 4, 107-118, 1985.

42. Hall, C. Rogers, W. and Barr, K. The use of imagery by athletes in selected sports. Sport Psychol. 4, 1-10, 1990.

43. Hanley, J. R, Young, A. W. and Pearson, N. A. Impairment of the visuo-spatial sketchpad. $Q . J . \exp$. Psychol. 43A, 101-125, 1991.

44. Housner, L. D. and Hoffman, S. Imagery and short term motor memory. In Psychology of Sport and Motor Behavior, G. C. Roberts and K. M. Newell (Editors). Human Kinetics Publ., Champaign, Illinois, 1979.

45. Ingvar, D. H. and Philipson, L. Distribution of the cerebral blood flow in the dominant hemisphere during motor ideation and motor performance. Ann. Neurol. 2, 230-237, 1977.

46. Isaac, A., Marks, D. F. and Russell, D. G. An instrument for assessing imagery of movement: The vividness of movement imagery questionnaire (VMIQ). J. Ment. Imag. 10, 23-30, 1986.

47. Jacobson, E. Electrophysiology of mental activities. Am. J. Psychol. 44, 677-694, 19 ?.

48. James, W. Psychology: A briefer course. Macmillan, London, 1892.

49. Jeannerod, M. The Brain Machine: The Development of Neurophysiological Thought. Harvard University Press, 1985.

50. Jeannerod, M. The representing brain: Neural correlates of motor intention and imagery. Brain Behav. Sci. 17, 187-245, 1994.

51. Johansson, G. Visual perception of biological motion and a model for its analysis. Percept. Psychophys. 14, 201-211, 1973.

52. Johnson, P. The functional equivalence of imagery and movement. $Q$. J. exp. Psychol. 34A, 349-365, 1982.

53. Kosslyn, S. M. Aspects of the neuroscience of visual cognition: Further developments. In Mental Images in Human Cognition, R. H. Logie and M. Denis (Editors), pp. 351-381. Elsevier, Amsterdam, 1991.

54. Kosslyn, S. M., Brunn, J. L., Cave, C. B. and Wallach, R. W. Individual differences in mental imagery ability: A computational analysis. Cognition 18, 195-244, 1984.

55. Laabs, G. J. Retention characteristics of different reproduction cues in motor short term memory. J. exp. Psychol. 100, 168-177, 1973.

56. Lee, D. and Young, D. S. Gearing action to the environment. In Generation and Modulation of Action Patterns, H. Heuer and C. Fromm (Editors), pp. 217-230. Springer, Berlin, 1986. 
57. Logie, R. H. and Baddeley, A. Imagery and working memory. In Imagery: Current Developments, P. J. Hampson, D. F. Marks and J. T. E. Richardson (Editors), pp. 105-115, London: Routledge, 1991.

58. Mackay, D. G. The problem of rehearsal or mental practice. J. Mot. Behav. 13, 274-285, 1981.

59. Mackay, D. G. The problem of flexibility, fluency and speed-accuracy trade-off in skilled behavior. Psychol. Rev. 89, 483-506, 1982.

60. Mahoney, M. J. and Avener, M. Psychology of the elite athlete: An exploratory study. Cognit. Res. Ther. 1, 135-141, 1977

61. Mahoney, M. J., Gabriel, T. J. and Perkins, T. S. Psychological skills and exceptional athletic performance. The Sport Psychologist 1, 181-199, 1987.

62. Marks, D. Imagery and consciousness: A theoretical review from an individual differences perspective. $J$. Ment. Imag. 2, 275-290, 1977.

63. McNeil, D. So you think gestures are non-verbal? Psychol. Rev. 92, 350-371, 1985.

64. Moody, D. L. Imagery differences among women of varying levels of experience, interests and abilities in motor skills. Res. Qrt. 38, 441-448, 1967.

65. Murphy, S. Models of imagery in sport: A review. J. Ment. Imag. 14, 153-172, 1990.

66. Newell, A. and Rosenbloom, P. S. Mechanisms of skill acquisition and the law of practice. In Cognitive Skills and their Acquisition, J. R. Anderson (Editor), pp. 1-55. Laurence Erlbaum, Hillsdale, New Jersey, 1981.

67. Orlick, T. Psyching for Sport: Mental Training for Athletes. Leisure Press, Champaign, 1986.

68. Paivio, A. Imagery and Verbal Processes. Holt, Reinhart \& Winston, New York, 1971.

69. Paivio, A. Cognitive and motivational functions of imagery in human performance. Can. J. appl. Sport Sci. 10, 4, 22 S-28 S, 1985.

70. Paivio, A. Mental Representations: A Dual Coding Approach. Oxford University Press, Oxford, 1986.

71. Paivio, A. and Clark, J. M. Static vs dynamic imagery. In Imagery and Cognition, C. Cornoldi and M. A. McDaniel (Editors), pp. 221-245. Springer, New York, 1991.

72. Paivio, A. and Harshman, R. Factor analysis of a questionnaire on imagery and verbal habits and skills. Can. J. Psychol. 37, 461-483, 1983.

73. Patrick, J. The effect of interpolated motor activities in short term motor memory. J. Mot. Behav. 3, 39-48, 1971.

74. Posner, M. I. and Raichle, M. E. Images of Mind. W. H. Freeman, San Francisco, 1994.

75. Reisberg, D. T. and Logie, R. The ins and outs of working memory: Overcoming the limits on learning from imagery. In Imagery, Creativity and Discovery: A cognitive approach, M. Intons-Peterson, B. RoskosEwoldson and R. Anderson (Editors), pp. 39-76. Elsevier, Amsterdam, 1993.

76. Richardson, A. Mental practice: A review and discussion. Res. Quart. 38, 95-107, 263-273, 1967.

77. Richardson, J. T. E Vividness and unvividness: Reliability, consistency and validity of subjective imagery ratings. J. Ment. Imag. 12, 115-122, 1988.

78. Richardson, J. T. E. Imagery and the brain. In Imagery and Cognition, C. Cornoldi and M. McDaniel (Editors), pp. 1-45. Springer, Berlin, 1991.

79. Roland, P. E., Larsen, B., Lassen, N. A. and Skinhoj, E. Supplementary motor area and other cortical areas in organisation of voluntary movements in man. J. Neurophysiol. 43, 118-136, 1980.

80. Rosenbaum, D. A. Motor programming: A review and scheduling theory. In Motor Behavior: Programming, Control and Acquisition, H. Heuer, U. Kleinbeck and K.-H. Schmidt (Editors), pp.1-33. Springer, Berlin, 1985.

81. Rotella, R. J., Gansneder, B., Ojala, D. and Billing, J. Cognition and coping strategies of elite skiers: an exploratory study of young developing athletes. J. Sport Psychol. 2, 350-354, 1980.

82. Sackett, R. S. The influence of symbolic rehearsal upon the retention of a maze habit. J. gen. Psychol. 10, 376-389, 1934.

83. Schmidt, R. A. A schema theory of discrete motor skill learning. Psychol. Rev. 82, 225-260, 1975.

84. Shaffer, L. H. Analysing piano performance: A study of concert pianists. In Tutorials in Motor Behavior, G. Stelmach and J. Requin (Editors), pp. 443-455.North Holland, Amsterdam, 1980.

85. Sheehan, P. W. A shortened form of Betts' Questionnaire upon Mental Imagery. J. clin. Psychol. 23, 386$389,1967$.

86. Shepard, R. N. The role of transformations in spatial cognition. In Spatial Cognition: Brain Bases and Development, J. Stiles-Davis, M. Kritchevsky and U. Bellugi (Editors). Laurence Erlbaum, Hillsdale, New Jersey, 1988.

87. Shepard, R. N. and Metzler, J. Mental rotation of three-dimensional objects. Science 171, 701-703, 1971.

88. Smyth, M., Pearson, N. A. and Pendleton, L. Movement and working memory: Patterns and positions in space. Q. J. exp. Psychol. 40A, 497-514, 1988.

89. Smyth, M. and Pendleton, L. Working memory for movements. Q. J. exp. Psychol. 41A, 235-250, 1989.

90. Smyth, M. and Pendleton, L. Space and movement in working memory. Q. J. exp. Psychol. 42A, 291-304, 1990.

91. Smyth, M. and Scholey, K. Interference in immediate spatial memory. Mem. Cognit. 22, 1-13, 1994. 
92. Stanislavski, C. (Translated E. R. Hapgood) Building a Character. Methuen, London, 1968.

93. Stelmach, G. E., Phillips, J. G. and Chau, A. W. Visuo-spatial processing in Parkinsonians. Neuropsychologia 27, 485-493, 1989.

94. Thorndike, E. L. Human Learning. Century, 1931. (Reprinted MIT Press, Cambridge, 1966.)

95. Ulich, E. Some experiments on the functioning of mental training in the acquisition of motor skills. Ergonomics 10, 411-419, 1967.

96. Ungeleider, S. and Golding, J. M. Mental practice among olympic athletes. Percept. Mot. Skills 72, 1007 $1017,19 ?$ ?

97. Vandenberg, S. G. and Kuse, A. R. Mental rotations: A group test of three- dimensional spatial visualisation. Percept. Mot. Skills 47, 599-601, 1978.

98. Whiting, H. T. A., Bijlard, M. J. and den Brinker, B. P. The effect of the availability of a dynamic model on the acquisition of a complex cyclical action. Q.J. exp. Psychol. 39A, 43-59, 1987.

99. Whiting, H. T. A. and den Brinkerk, B. P. Image of the act. In Theory and Research in Learning Disabilities, R. F. Mulcahy and A. E. Wall (Editors), pp. 217-235. Plenum Press, New York, 1982.

100. Williams, J. D., Riippon, G., Stone, B. and Annett, J. Psychophysiological correlates of dynamic imagery. (Submitted to Br. J. Psychol.)

101. Walshe, W. D., Russell, D. G. and Imanaka, K. Memory for movement: Interaction of location and distance cues and imagery ability. Acta Psychol. 44, 117-130, 1980. 